\title{
The AMIGA sample of isolated galaxies
}

\section{Far-infrared and radio continuum study of nuclear activity ${ }^{\star}$}

\author{
J. Sabater ${ }^{1}$, S. Leon ${ }^{2}$, L. Verdes-Montenegro ${ }^{1}$, U. Lisenfeld ${ }^{3}$, J. Sulentic ${ }^{4}$, and S. Verley ${ }^{5}$ \\ ${ }^{1}$ Instituto de Astrofísica de Andalucía, CSIC, Apdo. 3004, 18080 Granada, Spain \\ e-mail: jsm@iaa.es \\ 2 Instituto de RadioAstronomía Milimétrica (IRAM), Granada, Spain \\ 3 Dept. Física Teórica y del Cosmos, Universidad de Granada, Spain \\ 4 Department of Astronomy, Univ. of Alabama, Tuscaloosa, USA \\ 5 Osservatorio Astrofisico di Arcetri, Istituto Nazionale di Astrofisica, Firenze, Italy
}

Received 3 October 2007 / Accepted 29 February 2008

\section{ABSTRACT}

\begin{abstract}
Context. This paper is part of a series involving the AMIGA project (Analysis of the Interstellar Medium of Isolated GAlaxies). This project provides a statistically-significant sample of the most isolated galaxies in the northern sky.

Aims. We present a study of the nuclear activity in a well-defined sample of the most isolated galaxies (total sample: $n=1050$, complete subsample: $n=719$ ) in the local Universe traced by their far-infrared (FIR) and radio continuum emission.

Methods. We use the well-known radio continuum-FIR correlation to select radio-excess galaxies that are candidates to host an active galactic nucleus (AGN), as well as the FIR colours to find obscured AGN-candidates. We also used the existing information on nuclear activity in the Verron-Cetty catalogue and in the NASA Extragalactic Database.

Results. A final catalogue of AGN-candidate galaxies has been produced that will provide a baseline for studies on the dependence of activity on the environment. Our sample is mostly radio quiet, consistent with its high content of late-type galaxies. At most $\sim 1.5 \%$ of the galaxies show a radio excess with respect to the radio-FIR correlation, and this fraction even goes down to less than $0.8 \%$ after rejection of back/foreground sources using FIRST. We find that the fraction of FIR colour selected AGN-candidates is $\sim 28 \%$ with a lower limit of $\sim 7 \%$ Our final catalogue contains 89 AGN candidates and is publicly available on the AMIGA web page (http:// www.iaa.csic.es/AMIGA.html). A comparison with the results from the literature shows that the AMIGA sample has the lowest ratio of AGN candidates, both globally and separated into early and late types. Field galaxies as well as poor cluster and group environments show intermediate values, while the highest rates of AGN candidates are found in the central parts of clusters and in pair/merger dominated samples. For all environments, early-type galaxies show a higher ratio of radio-excess galaxies than late types, as can be expected, since massive elliptical galaxies are the usual hosts of powerful radio continuum emission.

Conclusions. We conclude that the environment plays a crucial and direct role in triggering radio nuclear activity and not only via the density-morphology relation. Isolated, early-type galaxies show a particularly low level of activity at radio wavelengths hence constituting the most nurture-free population of luminous early-type galaxies.
\end{abstract}

Key words. galaxies: evolution - galaxies: interaction - galaxies: active - surveys

\section{Introduction}

Galaxy evolution depends strongly on the environment. In particular, galaxy-galaxy interactions can induce nuclear activity by removing angular momentum from the gas and, in this way, feeding the central black hole. Hence, a higher rate of nuclear activity would be expected in interacting galaxies. However, different studies of this topic lead to contradictory results. Some works conclude that galaxies hosting an active galactic nucleus (AGN) have a higher number of companions than those with nonactive galactic nuclei (Petrosian 1982; Dahari 1985; MacKenty 1989, 1990; Rafanelli et al. 1995; Alonso et al. 2007), while others do not find this excess of interacting companions or find it only marginally (Bushouse 1986; Laurikainen \& Salo 1995; Schmitt 2001). Miller et al. (2003) find that the fraction of AGN is independent of the environment even in clusters.

* Full Tables 1, 2, 4-6 are available in electronic form at http://www.iaa.csic.es/AMIGA.html and at the CDS via anonymous ftp to cdsarc.u-strasbg. fr $(130.79 .128 .5)$ or via http://cdsweb.u-strasbg.fr/cgi-bin/qcat?]/A+A/486/73
Fuentes-Williams \& Stocke (1988) found only a marginal excess of similarly-sized galaxies but a clear excess of faint companions for Seyfert galaxies. Most recent works find a different result depending on the type of Seyfert galaxy (DultzinHacyan et al. 1999; Krongold et al. 2003). Recently Alonso et al. (2007) found for a sample of isolated galaxies a lower fraction of Type 2 AGN (23\%) than for close galaxy pairs (30\%). The proportion of galaxies hosting an AGN in extreme environments as compact groups was reported by Coziol et al. (2000) to be $70 \%$, while $50 \%$ was reported by Shimada et al. (2000). More recently Martinez et al. (2006a,b) studied a sample of 215 compact groups from the UZC (Updated Zwicky Catalog), and 42 galaxies belonging to compact groups from the Hickson Catalogue. They found in the first case $43 \%$ of AGN and $20 \%$ of transition objects (objects with spectroscopic properties between pure AGN and pure star forming), while in the second subset $57 \%$ showed characteristics consistent with lowluminosity AGN with a low-ionization nuclear emission-line region (LINER) type and 16\% were transition objects. 
Selection of AGN candidates using the radio-FIR correlation is also found in the literature. This correlation is very tight and can be used to distinguish galaxies for which their radio continuum emission is due to star formation and which follow the correlation, and those with an AGN causing an enhancement of the radio continuum emission that lie above the correlation. It is known that all AGN are radio sources at some level, e.g., Ho \& Ulvestad (2001) find that $85 \%$ of the nuclei of Seyfert galaxies are detected at radio wavelengths, with a wide range of intensities and morphologies (from compact cores to jet-like features). According to Reddy \& Yun (2004) a significant fraction of radio-excess objects are associated with luminous AGN. For their sample of 114 galaxies in nearby clusters, they find that $70 \%$ of the radio-excess galaxies are AGN based on different indicators, such as the presence of radio jets, X-ray emission, or optical emission lines. Reddy \& Yun consider this percentage as a lower limit of AGN among the radio-excess galaxies. They also analyse the sample of Miller \& Owen (2001) and find that $80 \%$ of the radio-excess galaxies in their sample of local Abell clusters are spectroscopically-confirmed AGN. Farinfrared (FIR) colours have also been demonstrated as useful to identify AGN candidates (de Grijp et al. 1985). We discuss studies based on these methods in more detail in Sect. 6 of this paper.

The contradictory results reached in previous studies might be due to the design of the surveys, sometimes focused on galaxies with emission lines, or due to different selection criteria of the samples. For example, although the sample in Alonso et al. (2007) was selected with a well-defined isolation criterion (i.e., no companions within a radius of $100 \mathrm{kpc}$ and a velocity difference of $350 \mathrm{~km} \mathrm{~s}^{-1}$ ) these parameters are not restrictive enough to ensure that a galaxy has been isolated for a significant fraction of its life. Hence, a well-defined sample of really isolated galaxies, which have remained isolated for a significant part of their life, is needed. The goal of the AMIGA project (Analysis of the interstellar Medium of Isolated GAlaxies, http: //www . iaa.csic.es/AMIGA.html; Verdes-Montenegro et al. 2005) is, therefore, to identify a statistically-significant sample of the most isolated galaxies in the local Universe and to quantify the properties of the interstellar medium in these galaxies and its relationship to the star formation and nuclear activity. In this paper, we will concentrate on the radio and FIR properties of this sample.

We define our sample of isolated galaxies in Sect. 2, and, in Sect. 3, we describe the data. Then we make use of different methods to select the AGN-candidates (Sect. 4). In Sect. 5, we present the final catalogue of AGN-candidates, and in Sect. 6, we discuss and compare our results to other studies from the literature. Finally, we present our conclusions in Sect. 7.

\section{The sample}

The starting sample for the AMIGA project is based on the Catalogue of Isolated Galaxies (CIG; Karachentseva 1973) which is composed of 1050 galaxies. In previous works we have: 1) revised all of the CIG positions (Leon \& Verdes-Montenegro 2003); 2) optically characterised the sample (Verdes-Montenegro et al. 2005); 3) performed a revision of the morphologies (Sulentic et al. 2006); 4) derived mid-infrared (MIR) and FIR basic properties (Lisenfeld et al. 2007); 5) performed a careful reevaluation of the degree of isolation of the CIG (Verley et al. 2007a,b); and 6) derived radio continuum properties (Leon et al. 2008).

In order to reduce biases in our statistical study we have used the completeness test $\left\langle V / V_{\mathrm{m}}\right\rangle$, as explained in
Verdes-Montenegro et al. (2005), and Lisenfeld et al. (2007). We adopted $m_{B}=15.0$ as the cutoff magnitude necessary to have a reasonably complete sample. This subsample contains 719 galaxies and in this paper we will refer to it as the "complete subsample", while the 1050 galaxies will be referred to as the "total sample". For studies that use IRAS satellite data, the total sample is reduced to 1030 galaxies while the complete subsample contains only 710 galaxies due to a number of galaxies falling in the region uncovered by IRAS known as the "IRAS gap" (see Lisenfeld et al. 2007). This difference in the number of galaxies does not affect the completeness of the subsample.

\section{The data}

We performed our study using archive data we reprocessed as well as data found in the literature.

\subsection{Observed and reprocessed data}

We obtained the FIR data for 1030 galaxies of the total sample by reprocessing the data of the IRAS satellite with the SCANPI tool (Lisenfeld et al. 2007). We obtained a better detection rate and an improved signal-to-noise level than in previous IRAS catalogues.

The radio continuum data has been obtained from two different sources: a) NRAO VLA Sky Survey (NVSS, $1.4 \mathrm{GHz}$; spatial resolution 45") and b) Faint Images of the Radio Sky at Twenty-cm (FIRST, 1.4 GHz; spatial resolution 5") as explained in detail in Leon et al. (2008). The radio continuum fluxes used in this paper were either taken from the NVSS survey catalogue or derived using the original survey data and extracting the flux with SExtractor (Bertin \& Arnouts 1996) within a radius of $35^{\prime \prime}$, obtaining in the latter case a higher detection rate. We use NVSS fluxes because of the high detection rate and sensitivity of this survey, which contains all galaxies belonging to our sample $(n=1050)$. In those cases where we found a radio enhancement (see Sect. 4.1.3), we complemented the NVSS data with the higher resolution images provided by FIRST. In this way, we improved the spatial location of the radio continuum emission to check whether this emission is due to a nuclear source or to a projected, unrelated source.

We used the distances given in Verdes-Montenegro et al. (2005) calculated with the Hubble constant $H_{0}=$ $75 \mathrm{~km} \mathrm{~s}^{-1} \mathrm{Mpc}^{-1}$.

We have computed the FIR and radio continuum luminosities with the following relations:

$$
\begin{aligned}
\log L_{1.4 \mathrm{GHz}}\left(\mathrm{W} \mathrm{Hz}^{-1}\right) & =20.08+2 \log D+\log S_{1.4 \mathrm{GHz}} \\
\log L_{60 \mu \mathrm{m}}\left(L_{\odot}\right) & =6.014+2 \log D+\log S_{60 \mu \mathrm{m}}
\end{aligned}
$$

where $D$ is the distance of the galaxy in Mpc and $S_{1.4 \mathrm{GHz}}$ and $S_{60 \mu \mathrm{m}}$ are the flux densities in Jy $\left(1 \mathrm{Jy}=10^{-26} \mathrm{~W} \mathrm{~m}^{-2} \mathrm{~Hz}^{-1}\right)$. The FIR luminosity ( $L_{\mathrm{FIR}}$; Helou et al. 1988) is related to the $L_{60} \mu \mathrm{m}$ by this formula:

$L_{\mathrm{FIR}}\left(L_{\odot}\right)=\left(1+\frac{S_{100 \mu \mathrm{m}}}{2.58 S_{60 \mu \mathrm{m}}}\right) L_{60 \mu \mathrm{m}}$.

The distribution of FIR luminosities for our sample peaks in $\log \left(L_{\text {FIR }} / L_{\odot}\right)=9.5-9.75$ (see Lisenfeld et al. 2007) and practically all galaxies have FIR luminosities between $\log \left(L_{\mathrm{FIR}} / L_{\odot}\right)=$ 7.5 and $\log \left(L_{\mathrm{FIR}} / L_{\odot}\right)=11.25$. The bulk of the FIR luminosities $(98 \%)$ lies below $\log \left(L_{\mathrm{FIR}} / L_{\odot}\right)=10.5$. 
Table 1. Galaxies from AMIGA sample listed as active in the literature ${ }^{1}$.

\begin{tabular}{cccc}
\hline \hline CIG $^{1}$ & NED $^{2}$ & V-C $^{2}$ & Our study $^{2,3}$ \\
\hline 6 & HII & & HII \\
33 & Sy? LINER & Sy? & \\
45 & HII & & HII \\
55 & LINER HII & & \\
56 & Sbrst & & Sbrst \\
57 & Sy2 & Sy2 & Sy2 \\
$\ldots$. & $\ldots$ & $\ldots$ & $\ldots$ \\
\hline
\end{tabular}

${ }^{1}$ Columns: 1) CIG number; 2) NED classification; 3) Véron-Cetty Catalogue classification; 4) Classification adopted for our study.

${ }^{2}$ Sy = Seyfert; HII = nuclear HII region; Sbrst= Starburst; SBNG $=$ small, bright nucleus galaxy; NLAGN $=$ narrow line active galaxies (a mixture of Seyfert 2's, LINERs, and starburst/AGN composites); DANS $=$ dwarf amorphous nuclei starburst galaxies.

${ }^{3}$ Classification obtained discarding those cases where a disagreement was found between the NED and Véron-Cetty classification.

\subsection{Data from the literature}

We have cross-correlated our sample with existing databases of active galaxies, in particular, the NASA Extragalactic Database $(\mathrm{NED})^{1}$ and the Véron-Cetty Catalogue of Quasars and Active Nuclei (Véron-Cetty \& Véron 2006, 12th edition).

The NED contains information on the type of nuclear activity for $n=77$ galaxies of our sample. The different classifications found in the sample are: LINER, HII, Starburst, DANS (dwarf amorphous nuclear starburst), SBNG (small, bright nucleus galaxy), NLAGN (narrow line AGN) and Seyfert. In some cases, the information on the Seyfert type is also given. The NLAGN are a mixture of Seyfert 2's, LINERs, and starburst/AGN composites. HII and starburst are not taken into account in our analysis since they are not considered non-stellar activity, but we list them in Table 1 . A total of $n=16$ galaxies from the AMIGA sample are classified as Seyferts, 1 as AGN and 5 as NLAGN in their catalogue.

The Véron-Cetty Catalogue of Quasars and Active Nuclei is a very complete compilation of active galaxies and quasars. It includes position and redshift as well as photometry $(U, B$, $V$ bands) and 6 and $21 \mathrm{~cm}$ flux densities when available. We have found $n=25$ of our galaxies in this catalogue. A total of $n=18$ are classified as Seyfert galaxies, 3 as HII, 3 as LINER and one has no assigned class. The 3 HII galaxies are included in this catalogue because in a previous version they were classified as Seyfert galaxies.

The LINERs are also known as Sy3 (Seyfert 3) in these catalogues. Although recent studies suggest some LINERs to be low-luminosity AGN (Ho 1999; González-Martín et al. 2006) this topic is not clear yet. In our study we consider LINERs separately from other kinds of active galaxies.

In Table 1 we list those galaxies found in the literature to show nuclear emissions. The first column is the CIG number, the second column is the type of emission found in NED, and the third column the one in the Véron-Cetty catalogue. In the fourth column, we indicate the classification taken for our study. It is obtained discarding those cases where a disagreement is found between the NED and Véron-Cetty classification.

${ }^{1}$ http://nedwww.ipac.caltech.edu/

\section{AGN selection methods}

Several methods to select AGN candidate galaxies exist. These different selection methods are generally biased toward a certain kind of nuclear activity. In this section, we discuss the results of applying selection criteria based on combined radio continuum and FIR data.

\subsection{Radio-FIR correlation}

The correlation between the FIR and the radio continuum emission is very tight and is attributed to star formation (Helou et al. 1985; Condon et al. 1991). Massive stars $\left(M \gtrsim 8 M_{\odot}\right)$ heat the dust which re-emits in the FIR. On the other hand, the supernovae produced at the end of the lives of these short-lived stars are ultimately responsible for the radio synchrotron emission from cosmic ray electrons accelerated in their shocks. Deviation from this correlation may be produced by nuclear activity (Sopp \& Alexander 1991; Niklas 1997; Yun et al. 2001; Drake et al. 2003), since those AGN with compact (200 pc) radio cores, i.e., some radio-quiet AGN and all radio-loud AGN (e.g., Impey \& Gregorini 1993; Roy et al. 1998) exhibit a radio excess.

Radio-loud active galaxies amount to approximately $10 \%$ of the optically-identified galaxies with an AGN (Kellermann et al. 1989; Hooper et al. 1995). In radio-loud AGN, the radio emission from the nucleus is added to the emission produced by star formation. If this additional emission is strong enough, an excess of radio emission will be found with respect to the radio-FIR correlation. We use the radio-FIR correlation to identify radio AGN candidates.

Since 468 galaxies in our complete subsample have at least one upper limit (radio or FIR) we have used survival analysis to compute the correlation to exploit the information carried in the upper limits. The Schmitt method (Schmitt 1985; Isobe et al. 1986) allows us to compute a correlation when upper limits exist in both the dependent and the independent variables. We use the complete subsample to calculate the correlation. We computed two regression lines to compare with other regressions of different samples and authors, one for $\log L_{1.4 \mathrm{GHz}}\left(\mathrm{W} \mathrm{Hz}^{-1}\right)$ versus $\log L_{60} \mu \mathrm{m}\left(L_{\odot}\right)$ and the other for $\log L_{1.4 \mathrm{GHz}}\left(\mathrm{W} \mathrm{Hz}^{-1}\right)$ versus $\log L_{\mathrm{FIR}}\left(L_{\odot}\right)$. The regression lines are:

$$
\begin{aligned}
\log L_{1.4 \mathrm{GHz}}\left(\mathrm{W} \mathrm{Hz}^{-1}\right)= & {[1.02 \pm 0.03] \log \left(L_{\mathrm{FIR}} / L_{\odot}\right) } \\
+ & {[11.4 \pm 0.3] } \\
\log L_{1.4 \mathrm{GHz}}\left(\mathrm{W} \mathrm{Hz}^{-1}\right)= & {[1.025 \pm 0.023] \log \left(L_{60 \mu \mathrm{m}} / L_{\odot}\right) } \\
+ & {[11.75 \pm 0.21] . }
\end{aligned}
$$

In both cases, the slope is close to one. These regression lines have been computed adopting $\log L_{1.4 \mathrm{GHz}}$ as the independent variable and minimising the residuals in the y-axis. This is the usual way of computing the radio-FIR correlation in the literature, which enables us to carry out comparisons. Since we are interested in the physical relation between the two variables we prefer to use a symmetric method, the bisector best-fit (Isobe et al. 1990). In this case the regression lines are:

$$
\begin{aligned}
\log L_{1.4 \mathrm{GHz}}\left(\mathrm{W} \mathrm{Hz}^{-1}\right)= & {[1.06 \pm 0.03] \log \left(L_{\mathrm{FIR}} / L_{\odot}\right) } \\
+ & {[11.1 \pm 0.3] } \\
\log L_{1.4 \mathrm{GHz}}\left(\mathrm{W} \mathrm{Hz}^{-1}\right)= & {[1.07 \pm 0.03] \log \left(L_{60} \mu \mathrm{m} / L_{\odot}\right) } \\
+ & {[11.31 \pm 0.23], }
\end{aligned}
$$

hence deviating slightly from linearity. 


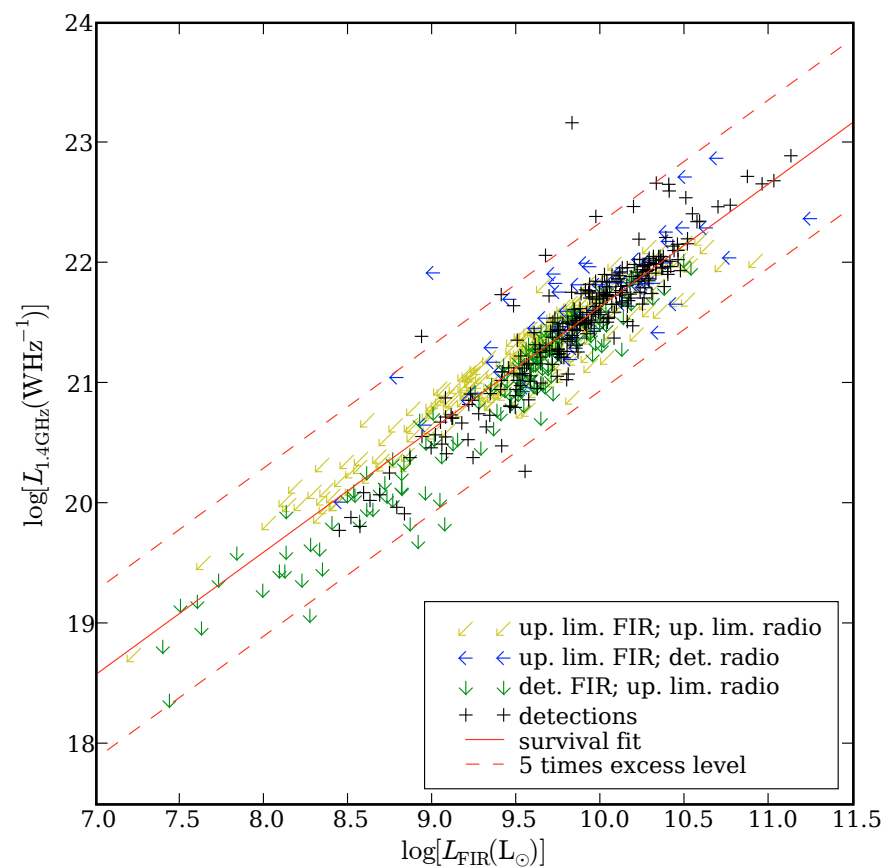

Fig. 1. Radio versus FIR luminosity for the complete subsample $(n=710)$. We show the correlation as a solid line and the 5 times radioexcess and FIR-excess levels as dashed lines. The galaxies above the upper-dashed line are the radio-excess galaxies.

\subsubsection{Radio-excess galaxies}

Radio-excess galaxies are defined, according to Yun et al. (2001), as those whose radio luminosity is larger than 5 times the value predicted by the radio-FIR correlation. In Fig. 1, we show the galaxies of our complete subsample with the regression fit plotted as a solid line. The dashed lines denote a deviation by a factor 5. Galaxies above the upper-dashed line are selected as radio-excess galaxies. There are 6 radio-excess galaxies in the complete subsample $(n=710)$ and 2 more in the total sample. Other studies (Niklas et al. 1995; Condon et al. 2002; Mauch \& Sadler 2007) use a cutoff factor of 3 for the radio excess, hence this cutoff will be also used to allow comparison with these samples. Using this value for our sample, we find 16 radio-excess galaxies in the complete subsample and 4 more in the total sample. In Table 2, we list the galaxies with a radio-excess (of a factor 3) for the total sample and, in Table 3, we detail separately the number of radio-excess galaxies and percentages for the complete subsample and for the early and late-type galaxies. The percentages are normalised to the number of galaxies that can be classified using this method. We can classify galaxies with detections in both bands, galaxies detected in radio continuum with an upper limit in FIR above the selection line and galaxies detected in FIR with an upper limit in radio continuum below the selection line.

None of the galaxies classified as Seyfert, LINER, NLAGN or AGN as listed in Col. 4 of Table 1 show a radio-excess with respect to the radio-FIR correlation by a factor 5 or more. We find 3 Seyfert galaxies located by more than a factor of 3 above the FIR-radio correlation: CIG 72, 692 and 877. CIG 72 has an upper limit in FIR and, according to Lim (priv. comm.), shows a tidal tail in HI linking it to a small companion. CIG 692 has been observed with the VLA in its A configuration at $8.3 \mathrm{GHz}$ by Schmitt et al. (2001) finding a symmetric triple source with a total extent of $3 \mathrm{kpc}$. CIG 877 is an elliptical galaxy studied by Marcum et al. (2004). They suggest that the IRAS emission
Table 2. Radio-excess galaxies found using the radio-FIR correlation.

\begin{tabular}{|c|c|c|c|c|c|}
\hline CIG & $\log L_{\mathrm{FIR}}^{1}$ & Code $^{2}$ & $\log L_{1.4 \mathrm{GHz}}^{3}$ & $\mathrm{~T}^{4}$ & Excess factor $^{5}$ \\
\hline \multicolumn{6}{|c|}{ Galaxies belonging to the complete subsample } \\
\hline 41 & 9.67 & 0 & 22.07 & $\mathrm{Sc}$ & 5.7 \\
\hline 72 & 9.72 & 1 & 21.91 & $\mathrm{Sbc}$ & 3.6 \\
\hline 97 & 10.19 & 0 & 22.47 & $\mathrm{Sc}$ & 4.3 \\
\hline 156 & 9.41 & 0 & 21.74 & SBab & 5.0 \\
\hline 187 & 9.83 & 0 & 23.17 & $\mathrm{Sc}$ & 50.2 \\
\hline 248 & 10.40 & 0 & 22.60 & Sc: & 3.5 \\
\hline 287 & 9.97 & 0 & 22.39 & SBbc & 6.0 \\
\hline 480 & 9.48 & 0 & 21.65 & SOa & 3.4 \\
\hline 488 & 10.50 & 1 & 22.72 & $\mathrm{Sb}$ & 3.7 \\
\hline 571 & 8.93 & 0 & 21.39 & $\mathrm{Sc}$ & 6.9 \\
\hline 591 & 9.46 & 1 & 21.39 & $\mathrm{Sbc}$ & 4.1 \\
\hline 692 & 10.33 & 0 & 22.67 & $\mathrm{Sb}$ & 4.9 \\
\hline 734 & 8.79 & 1 & 21.05 & S0a & 4.4 \\
\hline 877 & 10.40 & 0 & 22.66 & $\mathrm{E}:$ & 4.0 \\
\hline 893 & 10.69 & 1 & 22.87 & E/S0 & 3.4 \\
\hline 1045 & 8.78 & 1 & 21.92 & S0 & 19.7 \\
\hline \multicolumn{6}{|c|}{ Galaxies not belonging to the complete subsample } \\
\hline 57 & 10.23 & 1 & 22.73 & $\mathrm{Sb}$ & 5.6 \\
\hline 510 & 9.42 & 1 & 21.61 & $\mathrm{Sc}$ & 3.6 \\
\hline 836 & 10.17 & 1 & 23.29 & $\mathrm{E} / \mathrm{S} 0$ & 29.7 \\
\hline 999 & 9.90 & 0 & 22.09 & $\mathrm{Sa}$ & 3.5 \\
\hline
\end{tabular}

${ }^{1} \log L_{\mathrm{FIR}}$ from Lisenfeld et al. (2007); ${ }^{2}$ detection code for $\log L_{\mathrm{FIR}}$ : 0 for detections and 1 for upper limits; ${ }^{3} \log L_{1.4 \mathrm{GHz}}$ from Leon et al. (2008); ${ }^{4}$ morphological type from Sulentic et al. (2006), except for CIG 999 (Karachentseva 1973, NED); ${ }^{5}$ radio luminosity excess above the radio-FIR correlation.

Table 3. Radio-excess ratios for the complete subsample ${ }^{1}$.

\begin{tabular}{ccccccccc}
\hline \hline & \multicolumn{3}{l}{ Radio-excess galaxies } & \multicolumn{4}{c}{ FIRST revised $^{2}$} \\
\cline { 2 - 9 } Morpho $^{3}$ & \multicolumn{2}{l}{ Factor 5 } & \multicolumn{2}{c}{ Factor 3 } & \multicolumn{2}{c}{ Factor 5 } & \multicolumn{3}{c}{ Factor 3 } \\
\cline { 2 - 9 } & $\mathrm{N}$ & $\%$ & $\mathrm{~N}$ & $\%$ & $\mathrm{~N}$ & $\%$ & $\mathrm{~N}$ & $\%$ \\
\hline All T $(n=397)$ & 6 & 1.5 & 16 & 4.0 & 3 & 0.8 & 12 & 3.0 \\
E-S0a $(n=21)$ & 1 & 4.8 & 4 & 19.1 & 1 & 4.8 & 4 & 19.1 \\
Sa-Irr $(n=376)$ & 5 & 1.3 & 12 & 3.2 & 2 & 0.5 & 8 & 2.1 \\
\hline
\end{tabular}

${ }^{1}$ All percentages for the fraction of radio-excess galaxies are upper limits as explained in Sect. 4.1.3. ${ }^{2}$ In Sect. 4.1 .3 we explain how we revise these numbers using the FIRST survey. ${ }^{3}$ Morphological subsamples. The percentages are computed over the number of galaxies for each subsample.

assigned to this galaxy might be produced by two nearby stars. If this is true the radio excess would be even higher.

\subsection{2. q-parameter}

The $q$-parameter (Helou et al. 1985) is a good estimator of the deviation from the radio-FIR correlation. It has been found to be independent of the starburst strength (Lisenfeld et al. 1996) and distance (Yun et al. 2001). It is defined as:

$q \equiv \log \left[\frac{\mathrm{FIR}}{3.75 \times 10^{12} \mathrm{Wm}^{-2}}\right]-\log \left[\frac{S_{1.4 \mathrm{GHz}}}{\mathrm{Wm}^{-2} \mathrm{~Hz}^{-1}}\right]$

where $S_{1.4 \mathrm{GHz}}$ is the flux density at $1.4 \mathrm{GHz}$ in units of $\mathrm{Wm}^{-2} \mathrm{~Hz}^{-1}$ and FIR is the FIR flux calculated as:

$\mathrm{FIR} \equiv 1.26 \times 10^{-14}\left(2.58 S_{60 \mu \mathrm{m}}+S_{100 \mu \mathrm{m}}\right) \mathrm{Wm}^{-2}$

where $S_{60 \mu \mathrm{m}}$ and $S_{100 \mu \mathrm{m}}$ are IRAS $60 \mu \mathrm{m}$ and $100 \mu \mathrm{m}$ band flux densities in Jy. 


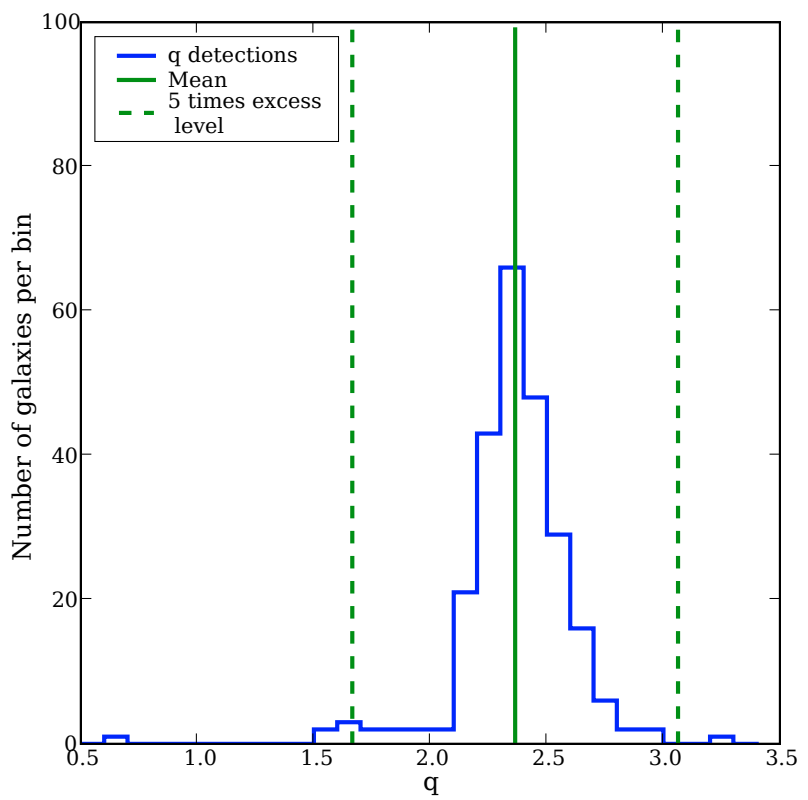

Fig. 2. Histogram of the $q$-parameter showing the galaxies of the complete subsample detected both in radio and infrared $(n=248)$. We also show the mean with a solid line and the 5 times radio-excess and FIRexcess limits with a dashed line. Radio-excess galaxies are located in the left tail of the histogram with lower $q$ values.

We have computed the $q$-parameter using only the galaxies detected both in FIR and in radio continuum $(n=248)$ because non-detections will result in upper and lower limits of the $q$-parameter and, as far as we know, there is no statistical method to take both of them into account simultaneously. In Fig. 2, a histogram of the $q$-parameter is shown. The mean value is 2.36 with a dispersion of $\sigma=0.24$. We define radio-excess galaxies in the same way as before by a deviation of more than a factor 5 , which translates into $q<1.66$. This condition is fullfilled for 4 out of 248 galaxies (CIG 41, 187, 287 and 571), corresponding to $1.6 \%$ of the studied galaxies. This rate is slightly higher than the one derived from the luminosity correlation $(\approx 1 \%)$, but we have to take into account that the errors are very high (small numbers) and that radio-excess galaxies probably have a higher chance of being detected in both radio continuum and FIR. So this fraction could be considered as an upper limit for the fraction of radioexcess galaxies.

\subsubsection{Projected radio sources}

Since the spatial resolution of the NVSS is not very high with respect to the size of the galaxies in our sample (97\% of them are smaller than $4^{\prime}$, cf. Lisenfeld et al. 2007) there is a chance that the radio excess found using the NVSS data is in fact due to a background/foreground source projected in the line of sight of the galaxy. We computed this probability with two methods. In the first method, we estimated the density of radio sources expected in the NVSS dividing the number of sources in the catalogue by the covered area, and obtained a value of 159825.4 sources/sr. Since we have searched for a NVSS source within a radius of $35^{\prime \prime}$ of each CIG galaxy, we determine that we could expect $n \approx 15$ unrelated sources in our total sample. In order to refine the previous value with a more local estimation of the probability, we estimated the average density of NVSS radio sources within a radius of $5^{\circ}$ of each CIG galaxy. The mean value found for the density is similar to the previous one within $10 \%$
Table 4. Radio-excess galaxies in FIRST.

\begin{tabular}{cccc}
\hline \hline CIG & NVSS flux (mJy) & FIRST flux $^{1}(\mathrm{mJy})$ & Distance $^{2}\left({ }^{\prime \prime}\right)$ \\
\hline 187 & 97.9 & 90.30 & 18.3 \\
248 & 14.3 & 10.24 & 0.5 \\
287 & 15.7 & 11.42 & 30.3 \\
480 & 6.1 & 5.56 & 0.3 \\
510 & 4.6 & 2.61 & 16.5 \\
571 & 16.3 & 13.88 & 36.9 \\
591 & 5.1 & 6.54 & 0.6 \\
734 & 3.3 & 2.11 & 26.8 \\
\hline
\end{tabular}

${ }^{1}$ Sum of FIRST fluxes within the NVSS search radius if more than one source is present; ${ }^{2}$ distance from the CIG galaxy centre to the FIRST source. Distance to the nearest FIRST source if more than one source is present.

(172561.7 sources/sr), hence implying $n \approx 16$ unrelated sources in our complete sample. The radio flux of a source projected in the field of a CIG galaxy would add to its normal emission and take it above the radio-FIR correlation. Since the expected number of unrelated sources is of the same order as the number of radio-excess galaxies, we expect an even lower ratio of galaxies above the radio-FIR correlation.

In the second method, we have made use of the higher spatial resolution of the FIRST survey to check whether the radio emission of the radio-excess galaxies is associated with the nucleus of the galaxy. Three among the 6 galaxies in the complete subsample with a radio excess larger than a factor 5 are covered by FIRST and all of them turn out to be sources unrelated to the nuclei. Seven of the 16 galaxies with a radio excess larger than a factor 3 in the same sample are covered by FIRST, and 4 of them are also unrelated sources. One of the 4 galaxies showing a radio excess not belonging to the complete subsample is covered by FIRST and we find its radio emission also to be separated from the nucleus. In Table 4, we list for all these sources the NVSS and FIRST fluxes and the distance between the nucleus and the closest FIRST source. NVSS and FIRST data were taken on different dates, but the fluxes in both surveys are very similar so that we can discount the possibility that the extranuclear emission is produced by a supernova. With these results, we have revised the percentages of radio-excess galaxies and give them in Table 3.

Since 5 out of the 8 galaxies revised with FIRST have proven to be projected sources and given the number of unrelated sources that we have estimated, there is a high probability that many of the remaining radio-excess galaxies are also unrelated sources. We computed the revised fractions of radio-excess galaxies considering all the galaxies without FIRST data as genuine detections, consequently the derived fractions (Table 3) should be considered as upper limits.

\subsection{IRAS colour}

In the work of de Grijp et al. (1985) a method to identify AGN candidates using FIR properties was presented. Galaxies hosting an AGN have, in general, a flatter spectrum in FIR. This is due to the warmer temperatures of the dust heated by the central engine. The advantage of the method is that it can identify obscured AGN that cannot be found with other wavelengths or methods. The success rate of this method is about $70 \%$. We select those galaxies with $S_{25 \mu \mathrm{m}} / S_{60 \mu \mathrm{m}} \geq 0.18$ as AGN-candidates, following the studies by Yun et al. (2001) and Reddy \& Yun (2004). 


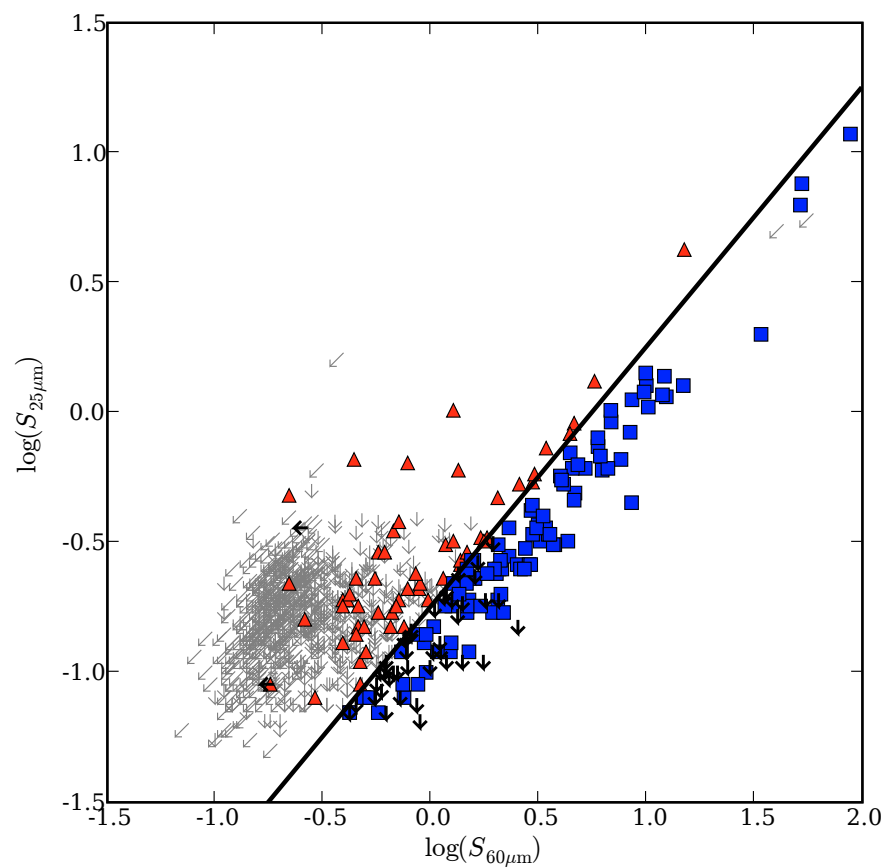

Fig. 3. Plot of $\log S_{25 \mu \mathrm{m}}$ versus $\log S_{60 \mu \mathrm{m}}$ for the total sample ( $n=$ 1030). The solid line corresponds to $S_{25 \mu \mathrm{m}} / S_{60 \mu \mathrm{m}}=0.18$. Galaxies classified as AGN-candidates lie above this line and are plotted as triangles and black left-arrows. Galaxies classified as non AGN-candidates are below the line, plotted with squares and black down-arrows. The remaining galaxies can not be classified due to upper limit in the fluxes (grey arrows).

Table 5. Classified galaxies using the IRAS colour method.

\begin{tabular}{cccccc}
\hline \hline CIG & ${\text { S25 }(\mathrm{Jy})^{1}}^{1}$ & Code $^{1,2}$ & S60 $^{(\mathrm{Jy})^{1}}$ & Code $^{1,2}$ & Classification $^{3}$ \\
\hline \multicolumn{5}{c}{ Galaxies belonging to the complete subsample } \\
4 & 0.61 & 0 & 5.19 & 0 & normal \\
41 & 0.19 & 0 & 0.39 & 0 & AGN \\
55 & 0.36 & 0 & 2.30 & 0 & normal \\
56 & 0.23 & 1 & 1.60 & 0 & normal \\
66 & 0.21 & 0 & 1.23 & 0 & normal \\
$\ldots$ & $\ldots$ & $\ldots$ & $\ldots$ & $\ldots$ & $\ldots$ \\
\hline \multicolumn{7}{c}{ Galaxies } & not belonging to the complete subsample \\
26 & 0.48 & 0 & 0.22 & 0 & AGN \\
62 & 0.25 & 0 & 2.07 & 0 & normal \\
80 & 0.92 & 0 & 6.84 & 0 & normal \\
105 & 0.66 & 0 & 7.60 & 0 & normal \\
121 & 0.60 & 0 & 6.22 & 0 & normal \\
$\ldots$ & $\ldots$ & $\ldots$ & $\ldots$ & $\ldots$ & $\ldots$
\end{tabular}

${ }^{1}$ Data from Lisenfeld et al. (2007); ${ }^{2}$ detection code: 0 for detections and 1 for upper limits; ${ }^{3}$ Normal for $S_{25 \mu \mathrm{m}} / S_{60 \mu \mathrm{m}}<0.18$ and AGN for $S_{25 \mu \mathrm{m}} / S_{60 \mu \mathrm{m}} \geq 0.18$.

In Fig. 3, we plot $\log S_{25} \mu \mathrm{m}$ versus $\log S_{60 \mu \mathrm{m}}$ for the total sample. The $S_{25} \mu \mathrm{m} / S_{60 \mu \mathrm{m}}$ ratio of 0.18 is plotted as a solid line. The triangles and squares denote detections and the arrows upper limits. A total of 197 galaxies of the total sample or 162 of the complete subsample can be classified using this method (Table 5) since they are: a) detections in both bands; b) detections at $S_{60 \mu \mathrm{m}}$ and upper limits at $S_{25} \mu \mathrm{m}$ with flux ratios below the $S_{25 \mu \mathrm{m}} / S_{60 \mu \mathrm{m}}=0.18$ line; or c) detections at $S_{25 \mu \mathrm{m}}$ and upper limits at $S_{60 \mu \mathrm{m}}$ with flux ratios above the $S_{25 \mu \mathrm{m}} / S_{60 \mu \mathrm{m}}=0.18$ line. Fifty-eight galaxies in the total sample and 46 in the complete subsample are AGN-candidates.

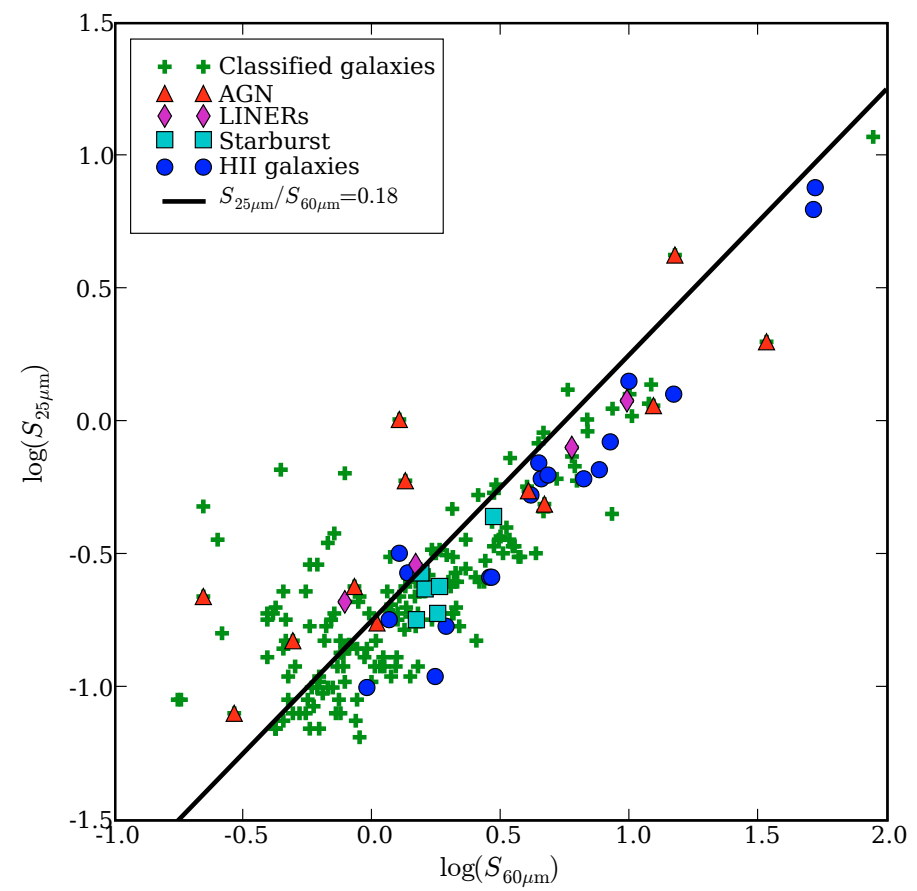

Fig. 4. Plot of $\log S_{25 \mu \mathrm{m}}$ versus $\log S_{60 \mu \mathrm{m}}$ for the CIG sample with available data about nuclear emission from the literature. The solid line corresponds to $S_{25 \mu \mathrm{m}} / S_{60 \mu \mathrm{m}}=0.18$. Galaxies that can be classified using this method are plotted as crosses $(n=197)$. AGN galaxies from the literature (triangles) are usually above the line. LINERs are plotted as diamonds. Most of the galaxies classified as HII and starburst in the literature (circles and squares respectively) are below the selection line.

Hence $28.4 \%$ of the galaxies for which a classification could be assigned are AGN-candidates. We obtained a lower limit to the total fraction of AGN-candidates of $6.5 \%$ by normalising to the complete subsample $(n=710)$. This is a lower limit because it assumes that the unclassified galaxies do not host an AGN.

Four of the radio-excess candidate galaxies can be also classified with the IRAS colour method and all of them (CIG 41, CIG 248, CIG 692 and CIG 877) are found to be AGN candidates. These galaxies have very warm IRAS colours with $S_{25 \mu \mathrm{m}} / S_{60 \mu \mathrm{m}}$ being higher than 0.45 in all cases.

Since for a number of CIG galaxies a detailed classification of their nuclear emission has been found in the literature (Sect. 3.2), we investigate specifically their location in the IRAS colour plot. This has allowed us to test the accuracy of the classification method. In Fig. 4, we plot the 197 galaxies that we were able to classify based on their IRAS colour and flag those galaxies classified as AGN, HII, LINER or starburst in the literature. A large fraction of the AGN galaxies are located above the selection cut $(7 / 12 \approx 60 \%)$, and almost all the HII and starburst galaxies (23/25) are below the line. This confirms our expectation that dust in AGN is usually warmer, hence, showing a higher $S_{25 \mu \mathrm{m}}$ to $S_{60 \mu \mathrm{m}}$ ratio while galaxies classified as HII should have lower values for that ratio. This result has no statistical significance, but shows the good agreement between the classification of their nuclear emission and their location in the $S_{25 \mu \mathrm{m}}$ versus $S_{60 \mu \mathrm{m}}$ plot.

\section{AGN catalogue}

We have compiled the results obtained from the methods explained in Sect. 4 as well as the data from the literature to produce a single catalogue of AGN-candidate galaxies for the total 
Table 6. Catalogue of AGN-candidates for the total sample ${ }^{1}$.

\begin{tabular}{cccccccc}
\hline \hline CIG & $\alpha(\mathrm{J} 2000)$ & $\delta(\mathrm{J} 2000)$ & Literature & FIR colour & $\begin{array}{c}\text { Radio-excess 3 } \\
\text { Factor 3 }\end{array}$ & $\begin{array}{c}\text { Radio-excess } \\
\text { Factor 5 }\end{array}$ & $\begin{array}{c}\text { Complete } \\
\text { subsample }\end{array}$ \\
\hline 26 & $00: 31: 52.85$ & $+37: 40: 43.2$ & - & AGN & 0 & 0 & 0 \\
41 & $00: 58: 23.36$ & $+36: 43: 50.2$ & - & - & 1 & 1 & 1 \\
44 & $01: 06: 35.60$ & $+10: 31: 18.1$ & - & AGN & 0 & 0 & 1 \\
57 & $01: 37: 48.25$ & $+02: 17: 27.3$ & Sy2 & - & 1 & 1 & 0 \\
69 & $01: 53: 42.23$ & $+29: 56: 01.5$ & - & AGN & 0 & 0 & 1 \\
$\ldots$ & $\ldots$ & $\ldots$ & $\ldots$ & $\ldots$ & $\ldots$ & $\ldots$ & $\ldots$ \\
\hline
\end{tabular}

${ }^{1}$ Columns: (1) CIG number; (2) $\alpha$ (J2000); (3) $\delta$ (J2000); (4) classification in the literature as listed in Col. 4 of Table 1; (5) AGN candidates using the FIR colour criterion; (6) 1 if is a radio-excess candidate galaxy using a factor 3 cutoff, 0 if not; (7) 1 if is a radio-excess candidate galaxy using a factor 5 cutoff, 0 if not; (8) 1 if the galaxy belongs to the complete subsample, 0 if not.

sample (Table 6). In the catalogue, we also indicate the galaxies composing the complete subsample. Since our confindence level varies as to the presence of an AGN depending on the selection method and its properties, as explained in the previous sections, we have included information on the method used.

The final catalogue is composed of:

- Active galaxies found in the NED and the Véron-Cetty catalogue (see Sect. 3.2). We have selected the 29 galaxies classified as Seyfert, LINER, NLAGN, or AGN as listed in Col. 4 of Table 1.

- Radio-excess candidates galaxies selected with the radioFIR correlation (see Sects. 4.1.1 and 4.1.3). We have included the 15 galaxies with at least a factor 3 radio excess using the $\log L_{1.4 \mathrm{GHz}}\left(\mathrm{W} \mathrm{Hz}^{-1}\right)$ versus $\log L_{\mathrm{FIR}}\left(L_{\odot}\right)$ correlation, taking into account the FIRST revision (Table 2).

- AGN-candidates selected with the IRAS colour criterion (see Sect. 4.2). We have added the 58 AGN-candidates selected using this method to the catalogue (Table 5).

\section{Discussion}

Based on the radio-FIR correlation we have determined a very low rate of AGN candidates, which further decreases after rejection of projected sources with FIRST data (Sect. 4.1.3). For the complete subsample, we find at most $0.8 \%$ of radio-excess galaxies with a factor 5 cutoff (or less than $4.0 \%$ for a factor 3 cutoff; Table 3 ). The AGN candidate rate based on the FIR colour selection is $\sim 28 \%$ (with a lower limit of $\backsim 7 \%$ ), higher than the radio-excess ratio as expected, since the colour method is sensitive to all types of AGN and not only to radio-loud objects. The optical luminosities of the galaxies with a radio excess have the same mean value as the complete sample, and the same occurs for FIR luminosities, although the results are only illustrative due to their low number. A trend is found for radio excess galaxies to have earlier morphological types, particularly in the subset showing a radio-excess above the factor 3 cutoff: $25 \%$ of the galaxies with radio-excess have morphologies earlier than $\mathrm{Sa}$, while for the complete sample the percentage of early types is $12 \%$.

Our sample is mostly radio quiet, with only a few galaxies $\left(1.3 \%\right.$ ) above a radio power of $10^{23} \mathrm{~W} \mathrm{~Hz}^{-1}$, consistent with the high ratio of late-type galaxies, which are usually radio quiet, in our sample. The only galaxy with a radio power above $10^{23} \mathrm{~W} \mathrm{~Hz}^{-1}$ in the complete subsample was found, based on FIRST data, to be an unrelated source (CIG 187). The radio continuum emission is dominated by mild star-formation (Leon et al. 2008), in contrast to a sample dominated by radio-AGN, the 2dF Galaxy Redshift Survey (2dFGRS; Sadler et al. 2002), where $60 \%$ of the galaxies are classified as AGN, and $75 \%$ have a radio power at $1.4 \mathrm{GHz}$ higher than $10^{23} \mathrm{~W} \mathrm{~Hz}^{-1}$. The CIG presents at most $30 \%$ of the total radio power density at $1.4 \mathrm{GHz}$ of the star-forming sample of Yun et al. (2001) or $10 \%$ of AGN-dominated samples (Sadler et al. 2002; Condon et al. 2002) in the local Universe. This low level of radio continuum emission at $1.4 \mathrm{GHz}$ for the CIG is confirmed by the low radio-to-optical ratio compared to emission-line galaxies (KISS sample; Van Duyne et al. 2004) as shown in Leon et al. (2008).

\subsection{Comparison with other samples}

Studies of the radio power and FIR emission of galaxies have been performed mostly for two kinds of samples: those referred to as field galaxies in the literature (e.g., Condon \& Broderick 1991; Yun et al. 2001; Miller \& Owen 2001; Corbett et al. 2002; Condon et al. 2002; Drake et al. 2003) where usually no environmental selection criterion has been applied, and cluster samples (e.g., Niklas et al. 1995; Andersen \& Owen 1995; Miller \& Owen 2001; Reddy \& Yun 2004; Omar \& Dwarakanath 2005). We summarise the main results of these papers in Tables 7 and 8.

We notice that a detailed comparison of these papers is difficult for a number of reasons that we list below. The selection criteria of the samples differ significantly, introducing different biases depending on whether the selection was performed at optical wavelengths or with a FIR or radio cut selection criterion. Normalisation of the percentages is also a delicate issue since, in some cases, the values are divided by the total number of galaxies with available data, but in others only detections are used. In the cases where AGN selection was performed using the radioFIR correlation, the wavelength of the radio-emission is usually $1.4 \mathrm{GHz}$ but in some cases it is $4.8 \mathrm{GHz}$. The radio excess cutoff varies for different papers, the most used factors are 3 times or 5 times above the radio-FIR correlation. Finally, the used radioFIR correlation is, in some cases, the one obtained for the sample analysed in the paper, but in others the one for a different reference sample is used, e.g., Omar \& Dwarakanath (2005) used Yun et al. (2001) correlation.

We have obtained the figures given in Table 7 by reanalysing the data given in the papers (e.g. most of them had not performed an analysis by morphological type) or provided by the authors, in an attempt to homogenise the statistics. We have defined the radio excess in two ways: a factor 3 or a factor 5 above the radioFIR correlation for our sample of isolated galaxies, corresponding to $q<1.88$ or $q<1.66$, respectively. The percentages are calculated with respect to the total number of galaxies that can be classified as explained in Sect. 4.1.1. The rates for different 
Table 7. Rate of radio-excess galaxies in the literature.

\begin{tabular}{|c|c|c|c|c|c|c|c|c|c|c|c|}
\hline \multirow[b]{3}{*}{ Sample } & \multirow[b]{3}{*}{ Environment } & \multicolumn{9}{|c|}{ Rate of Radio-excess galaxies 1} & \multirow[b]{3}{*}{ Notes } \\
\hline & & \multicolumn{3}{|c|}{ Total } & \multicolumn{3}{|c|}{ E-SOa } & \multicolumn{3}{|c|}{ Sa-Irr } & \\
\hline & & $\mathrm{N}^{2}$ & $\mathrm{~F}^{3}$ & $\mathrm{~F}^{3}{ }^{3}$ & $\mathrm{~N}^{2}$ & $\mathrm{~F}^{3}{ }^{3}$ & $\mathrm{~F}^{3}{ }^{3}$ & $\mathrm{~N}^{2}$ & $\mathrm{~F} 5^{3}$ & $\mathrm{~F}^{3}{ }^{3}$ & \\
\hline $\mathrm{AMIGA}^{4}$ & Isolated & 397 & 0.8 & 4.0 & 21 & 4.7 & 19.1 & 376 & 0.5 & 2.1 & $m_{B}<15$ \\
\hline Condon (1991) & - & 122 & 32.0 & 33.6 & 11 & 90.9 & 90.9 & 71 & 9.9 & 12.7 & $\begin{array}{c}S_{60 \mu \mathrm{m}}>0.2 \mathrm{Jy} \\
\text { and } S_{4.85 \mathrm{GHz}}>25 \mathrm{mJy}\end{array}$ \\
\hline Yun (2001) & - & 1809 & 1.3 & - & - & - & - & - & - & - & $S_{60 \mu \mathrm{m}}>2 \mathrm{Jy}$ \\
\hline Corbett (2002) & - & 82 & 2.4 & 4.9 & - & - & - & - & - & - & $S_{60 \mu \mathrm{m}}>4 \mathrm{Jy}$ \\
\hline Condon (2002) & - & 1897 & 8.2 & 10.8 & 287 & 42.2 & 51.6 & 1498 & 2.1 & 3.4 & $\begin{array}{l}S_{1.4 \mathrm{GHz}}>2.5 \mathrm{mJy} \\
\quad \text { and } m_{\mathrm{P}}<14.5\end{array}$ \\
\hline Drake (2003) & - & 178 & 55.1 & 60.7 & - & - & - & - & - & - & $\begin{array}{l}S_{4.8 \mathrm{GHz}} \gtrsim 16 \mathrm{mJy} \\
\text { and } S_{60 \mu \mathrm{m}} \gtrsim 0.1 \mathrm{Jy}\end{array}$ \\
\hline Omar (2005) & Eridanus group & 72 & 2.8 & 2.8 & 20 & 5.0 & 5.0 & 46 & 2.2 & 2.2 & \\
\hline Niklas (1995) & Virgo cluster & 37 & - & 16.2 & 2 & - & 0.0 & 35 & - & 17.1 & radio@4.8 GHz \\
\hline \multirow[t]{2}{*}{ Andersen (1995) } & Cluster \& group (poor) & 23 & 8.7 & 21.7 & - & - & - & - & - & - & \\
\hline & Cluster \& group (rich) & 20 & 15.0 & 25.0 & - & - & - & - & - & - & \\
\hline \multirow[t]{3}{*}{ Miller (2001) } & Clusters $0<r<1 \mathrm{Mpc}$ & 120 & 28.3 & 37.5 & 54 & 46.3 & 53.7 & 53 & 3.8 & 17.0 & $S_{1.4 \mathrm{GHz}}>10 \mathrm{mJy}$ \\
\hline & Clusters $1<r<2 \mathrm{Mpc}$ & 96 & 21.9 & 29.2 & 23 & 60.9 & 73.9 & 50 & 6.0 & 10.0 & $S_{1.4 \mathrm{GHz}}>10 \mathrm{mJy}$ \\
\hline & Clusters $2<r<3 \mathrm{Mpc}$ & 94 & 6.4 & 12.8 & 19 & 26.3 & 31.6 & 47 & 0.0 & 4.3 & $S_{1.4 \mathrm{GHz}}>10 \mathrm{mJy}$ \\
\hline \multirow[t]{3}{*}{ Reddy (2004) } & X-ray clusters & 114 & 13.2 & 19.3 & 33 & 30.3 & 45.5 & 81 & 6.2 & 8.6 & $L_{60 \mu \mathrm{m}}>8.92 L_{\odot}$ \\
\hline & $\mathrm{X}$-ray clusters core & 33 & 24.2 & 39.4 & 15 & 40.0 & 66.7 & 18 & 11.1 & 16.7 & $L_{60 \mu \mathrm{m}}>8.92 L_{\odot}$ \\
\hline & $\mathrm{X}$-ray clusters ring & 81 & 8.6 & 11.1 & 18 & 22.2 & 27.8 & 63 & 4.8 & 6.3 & $L_{60 \mu \mathrm{m}}>8.92 L_{\odot}$ \\
\hline
\end{tabular}

${ }^{1}$ The percentages are computed over the number of galaxies for each morphological subsample; ${ }^{2}$ number of galaxies in the total samples or the morphological subsamples; ${ }^{3} \mathrm{~F} 3$ : factor 3 radio excess; F5: factor 5 radio excess. Figures given in percentages; ${ }^{4}$ all percentages for the fraction of radio-excess galaxies are upper limits as explained in Sect. 4.1.3.

Table 8. Rate of AGN candidates in the literature from FIR colour.

\begin{tabular}{lclc}
\hline \hline & & \multicolumn{2}{c}{ Rate of AGN-candidates } \\
Sample & Environment & Total & 2 Jy cutoff \\
\hline AMIGA & Isolated & 28.4 & 14.3 \\
Condon 1991 & - & 21.8 & 12.4 \\
Yun 2001 & - & 15.9 & 15.9 \\
Condon 2002 & - & 13.3 & - \\
Drake 2003 & - & 45.0 & 22.6 \\
Andersen 1995 & Cluster \& group (poor) & 56.5 & - \\
& Cluster \& group (rich) & 75.0 & - \\
Reddy 2004 & X-ray clusters & 59.0 & 20.0 \\
\hline
\end{tabular}

${ }^{1}$ Figures given in percentages.

morphological types are not calculated with respect to the total number of galaxies but normalised for the corresponding subsamples of early and late-type galaxies. Comparison of the AGN candidate rate based on the FIR colour selection was possible for some of the references given above (Condon \& Broderick 1991; Yun et al. 2001; Drake et al. 2003; Andersen \& Owen 1995; Reddy \& Yun 2004). In these studies, $S_{25} \mu \mathrm{m}$ and $S_{60 \mu \mathrm{m}}$ fluxes or $\alpha_{25,60}$ (spectral index between $S_{25} \mu \mathrm{m}$ and $S_{60 \mu \mathrm{m}}$ ) were given, and we selected as AGN candidates those with $S_{25 \mu \mathrm{m}} / S_{60 \mu \mathrm{m}} \geq$ 0.18 (see Sect. 4.2) equivalent to $\alpha_{25,60}<1.958$. We have to be careful when comparing these numbers, since, e.g., the sample of Yun et al. (2001) has been selected to have $S_{60 \mu \mathrm{m}} \geq 2 \mathrm{Jy}$. This will bias the result since the AGN candidate rate depends directly on this variable. However, we think that this cut has a significant advantage since much of the AGN candidates have low IRAS fluxes (Fig. 3) near the detection limit. Therefore, the error is very high in comparison to the measurements, hence confidence in the selection of AGN candidates gets lower. For this reason, we have both provided the statistics using the original data in each paper and applying the $S_{60 \mu \mathrm{m}} \geq 2$ Jy cut to all samples when it was possible, including ours (see Table 8).

\subsection{1. "Field" environments}

As indicated above some studies of radio-excess galaxies were performed for samples usually referred to as "field" in the sense that they were not selected in any particular way with respect to their environment. However, it is found that, for instance, many cD galaxies belong to these "field" samples. We comment below on these studies.

Condon \& Broderick (1991) studied a sample of IRAS sources applying a high radio flux cutoff at $4.75 \mathrm{GHz}$ and with no definition of the environment (e.g., several pairs are included in their sample). They also apply a FIR cutoff but not as strict as the radio one. Using their data in Table 2 we have calculated the ratios of radio-excess listed in our Table 7 and that of AGN candidates based on $\alpha_{25,60}$ as given in Table 8 . When the $2 \mathrm{Jy}$ cut is applied to their sample the percentage of galaxies with $\alpha_{25,60}<1.958$ decreases, as expected. The radio flux cut leads also to a bias since it will increase the ratio of radio-excess galaxies with respect to other samples listed in Table 7, selected without such a cutoff or using a higher FIR cutoff, the latter obviously producing the opposite bias. The separation of the data by morphological types clearly shows a higher rate of radio excess for early-type galaxies.

Yun et al. (2001) studied in detail an IR flux-limited complete sample with no selection concerning the environment. Their fit to the radio-FIR correlation is identical to ours within the errors since both samples are dominated by star formation activity, with very few galaxies deviating from the correlation, hence leading to very similar $q$-parameters, 2.34 in their sample versus 2.36 in ours. Their ratio of radio-excess galaxies being very low, is still a factor 3 larger than ours. Two main differences are found between both samples. While our sample is optically selected, Yun et al. selected their sample in the FIR. Applying the same FIR flux cutoff to our complete subsample leads to a decrease of the ratio of radio-excess galaxies to $0 \%$. On the other hand, galaxies in their sample lie in higher density environments 


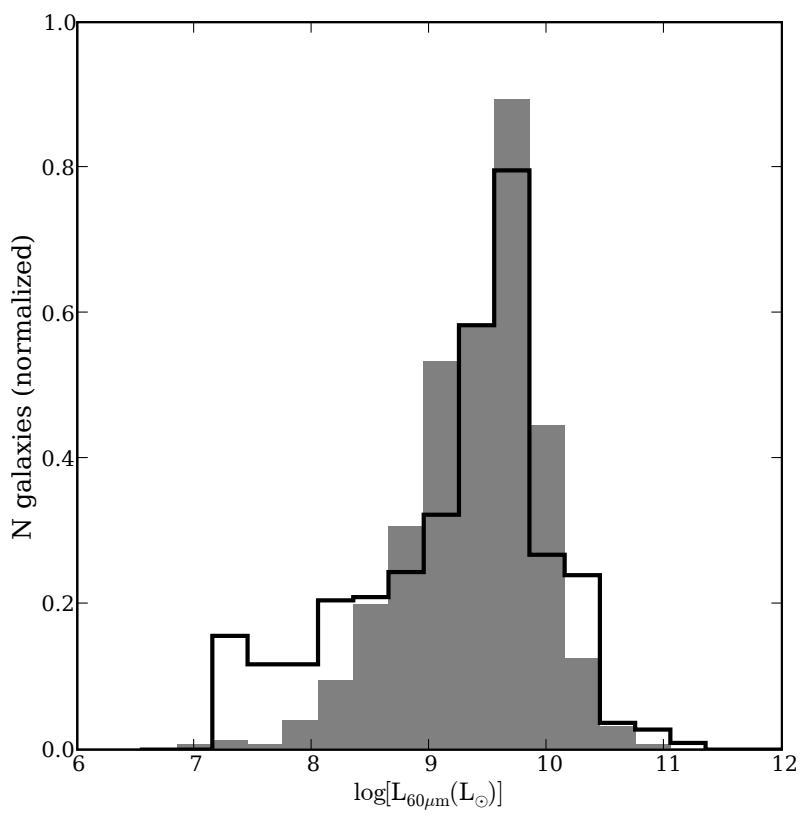

Fig. 5. Histogram of $L_{60} \mu \mathrm{m}$ luminosity for our complete subsample ( $n=$ 710; black line) and the Yun et al. (2001) sample ( $n=1248$; grey bars).

than ours: among the 23 radio excess galaxies in their sample 2 are giant E/SO in clusters and 16 galaxies are interacting in pairs or higher ranked environments. Furthermore, a comparison of the $\log L_{60 \mu \mathrm{m}}\left(L_{\odot}\right)$ distribution for both samples (Fig. 5) shows a higher ratio of galaxies at the low luminosity end for the AMIGA sample, with a difference in the mean of 0.31 . Since the FIR luminosity is a variable widely known to be driven by interaction this supports further the higher density of the environment traced by the sample in Yun et al. (2001). Unfortunately, no morphological types are given for their full sample and we could not check the percentages for early and late-type galaxies. They kindly provided the $S_{25} \mu \mathrm{m}$ and $S_{60 \mu \mathrm{m}}$ fluxes and we used them to derive the statistics of AGN candidates based on IRAS colours for their sample (Table 8).

Corbett et al. (2002) studied a sample of objects with IRAS data to perform a comparison of the multiwavelength properties of a sample of AGN with and without compact radio cores and a matched sample of galaxies without an AGN. They imposed a FIR flux limit higher than Yun et al. (2001). As a consequence, the Corbett et al. selected galaxies have FIR luminosities greater than $10^{10.5} L_{\odot}$ which, as they indicate, results in an enhanced fraction of interacting galaxies in the sample. They estimate that $40 \%$ of their sample is obviously involved in either tidal interactions or are major mergers, and $20 \%$ are apparently noninteracting. Using the data in Table 2 of Corbett et al. (2002), we obtained their ratio of radio-excess galaxies. We found a slightly higher value of radio-excess galaxies than for the sample of Yun et al. (2001); the Corbett et al. (2002) data yielded 3 times our value, but is still only a few percent. This low value is, as they indicate, most likely due to their high FIR cutoff, artificially lowering the rate of radio-excess galaxies.

Condon et al. (2002) studied an Uppsala General Catalogue of Galaxies (UGC) sample composed of sources detected in the NVSS and covered by the IRAS survey, using a radio-flux cutoff and no selection in terms of the environment. Using the data in their Table 1, we have calculated their rate of radio-excess galaxies, both total and separated by morphological types, as well as the percentage of AGN candidates with $\alpha_{25,60}<1.958$. We could not apply a cut at $S_{60 \mu \mathrm{m}} \geq 2$ Jy since $S_{60 \mu \mathrm{m}}$ was not available.
We note that the radio cutoff for this sample is lower than in Condon \& Broderick (1991), and the ratio of radio-excess galaxies is also lower. As in Condon \& Broderick (1991) a higher rate of radio excess is found for early-type galaxies.

Drake et al. (2003) used a sample selected by crosscorrelating the IRAS Faint Source Catalogue with the $5 \mathrm{GHz}$ Parkes-MIT-NRAO catalogue. We derived the ratio of AGN candidates with $S_{25 \mu \mathrm{m}} / S_{60 \mu \mathrm{m}} \geq 0.18$ using the data in their Table 1 and list the value with and without the $2 \mathrm{Jy}$ cut in Table 8 . They find a radio-excess rate of $\approx 55 \%$, using a factor 5 cutoff at $4.8 \mathrm{GHz}$ with respect to the mean of the $u$ parameter in Condon et al. (1995) $\left(u \equiv \log \left(S_{60 \mu \mathrm{m}} / S_{4.8 \mathrm{GHz}}\right) ;\langle u\rangle=2.5\right)$. They sample lower IRAS flux densities than previous authors and thus find a larger fraction of objects with intermediate radio fluxes. In a later paper (Drake et al. 2004) the host galaxies of radio-excess sources are studied, finding that only $23 \%$ of them are isolated and with no evidence of disturbance. They also determine the morphology of the hosts, obtaining that elliptical galaxies show a large range of radio excess, while the disk systems all have only a moderate radio excess.

\subsubsection{Dense environments}

Niklas et al. (1995) analysed the radio-FIR correlation for Virgo cluster galaxies at 4.8 and $10 \mathrm{GHz}$. Inspection of their Fig. 4 indicates that they used a cutoff of a factor 3 to select radioexcess galaxies with respect to their radio-FIR correlation. We have used the data in their paper to obtain the remaining values given in Table 7 .

Andersen \& Owen (1995) compared the FIR-radio correlation for spirals in rich and poor cluster and group environments. Using the data in their Tables 4 and 5 we calculated the radioexcess ratio in their subsample.

Miller \& Owen (2001) considered a sample of nearby Abell clusters galaxies. They kindly provided the data used in their paper which we have used to estimate the ratio of radio-excess galaxies in their sample for different distances from the cluster center, as we list in Table 7. They considered 3 subsamples: one including galaxies in the inner Mpc of clusters, one in the 1-2 Mpc range and the last one at the periphery of clusters, between 2 and $3 \mathrm{Mpc}$ from the cluster core in projection. The last subsample is likely to be very similar to field-like environments. They find a considerable gradient in the ratio of radio-excess galaxies from the outer parts to the centre of the cluster (see Table 7). They suggest that this is probably a result of the presence of centrally concentrated radio-luminous elliptical galaxies. No data on the morphological types are given in the paper. In order to investigate whether the same trend holds for spiral galaxies, we compiled the morphologies from NED. For the low density parts of Abell clusters we find a very low ratio of spirals with radio-excess and a much higher one for early types. For the inner $2 \mathrm{Mpc}$ the ratio of radio-excess galaxies increases by a factor 2-3 both for early and late-type galaxies.

Reddy \& Yun (2004) studied a sample of cluster galaxies located in the seven nearest clusters with prominent X-ray emission using a cut in the FIR luminosities. They use $q<1.64$ as cutoff for radio-excess galaxies, very similar to our value (1.66) but for coherence we recalculated the ratios referred to our value. We also estimated the ratios for early and late-type galaxies using the data in their Table 2. Their ratios are similar to the values in other clusters, except for those cases where a radio flux cutoff was applied, in which case the radio-excess ratios are higher due to selection effects. We provide also the ratios for galaxies with projected cluster-centric distances lower than $0.5 \mathrm{Mpc}$ 
and between 0.5 and $1.5 \mathrm{Mpc}$, defined by them as core and ring respectively. These data are not given in their paper and were kindly provided by the authors. We notice that, when divided into early and late-type galaxies, both early and late types show a significant increase in the ratio of radio-excess for smaller radii. This result cannot be directly compared with Miller \& Owen (2001) since the area defined as ring by Reddy \& Yun (2004) is still in the inner area of the Miller \& Owen sample. We used the $S_{25 \mu \mathrm{m}}$ and $S_{60 \mu \mathrm{m}}$ fluxes provided by them to derive the statistics of AGN candidates based on IRAS colours for their sample (Table 8).

Omar \& Dwarakanath (2005) studied the radio-FIR correlation for the Eridanus group, which is not dynamically relaxed. They consider the 72 galaxies detected in the IRAS survey. Using the data in their Fig. 3 and Table 2 we have calculated the radio excess for the total sample and divided into morphological types.

\subsubsection{Radio-excess, environment and density-morphology relation}

A comparison with the above discussed samples from the literature shows that the AMIGA sample has the lowest ratio of radioexcess galaxies, both globally and separated into early and late types (Table 7). Higher ratios of radio excess galaxies are found in denser but still poor environments as the outer parts of clusters with prominent X-ray emission (Reddy \& Yun 2004), outer parts of Abell clusters (Miller \& Owen 2001), poor cluster and group environment (Andersen \& Owen 1995), Virgo cluster (Niklas et al. 1995) and Eridanus group (Omar \& Dwarakanath 2005). The values in the outermost, field-like sample of Miller \& Owen are similar to the ones in Condon et al. (2002), suggesting that the outer parts of clusters are as efficient as the "field" environment to trigger radio emission in active galaxies. For the densest environments an even higher ratio of radio-excess is found, as in the core sample of Reddy \& Yun, the inner area of Abell clusters (Miller \& Owen 2001) or rich environments (Andersen \& Owen 1995). It is interesting to note that the Drake et al. sample is dominated by one-to-one interactions, i.e., higher local density, and its radio-excess rate is the highest among all. Although the radio-flux cutoff used in their sample is high, hence producing an artificial increase in radio-excess galaxies, the result might also reflect that one-to-one interactions are more efficient in inducing radio activity in galaxies than the larger scale environment. This could be explained by the fuelling of gas towards the centre of galaxies in interacting pairs, feeding the AGN and leading to the radio-excess regime.

The same result is found using FIR colours, independently whether the $2 \mathrm{Jy}$ cut is applied or not: we find that the rate of AGN candidates based on IRAS colours increases from the most isolated environments to the densest ones. We notice that when the $2 \mathrm{Jy}$ cut is applied, although the trend is kept the gradient is significantly reduced, confirming the limited reliability of the method.

Finally, we have studied several subsets of the AMIGA sample, taking into account the refinement of the CIG with respect to the environment that our group carried out. In our reevaluation of the optical morphologies of the CIG galaxies we identified 32 objects candidates to be suffering interactions based on evidence for asymmetries/distortions that might be of tidal origin (Sulentic et al. 2006). None of these galaxies show a radio excess above a cutoff of 3 . A further revision of the sample showed that 150 additional galaxies lie in environments that could affect their evolution based on the value of their local number density of neighbours and the tidal forces at play (Verley et al. 2007a). These galaxies are uniformly distributed with respect to the radio-FIR correlation suggesting that weak interactions in a low density environment do not significantly affect the radio activity of galaxies.

The higher ratio of radio-excess galaxies in denser environments has often been explained as due to the densitymorphology relation. In all the samples studied in this paper, a larger ratio of radio-excess galaxies is found for early types, which is not surprising since usually the typical host galaxies of an AGN with powerful radio continuum emission are massive ellipticals. The higher abundance of early-type galaxies in denser environments could by itself justify the higher ratio of radio active galaxies. We think however that although it might explain in part the results, the environment also has to play a prominent role in triggering the radio activity. On one hand the ratio of early-type galaxies with radio-excess in our sample, higher than for late-type galaxies, is still much lower than for all other environments (only $4.8 \%$ of the early-type galaxies have a radio excess above a factor 5 cutoff). This result might be explained by the low luminosities of the AMIGA early-type population relative to the AMIGA spiral population and to early-type populations found in most surveys (Sulentic et al. 2006). This shows that the low ratio of radio-excess galaxies in our sample can not be only due to the small percentage of early-type galaxies $(12 \%$ in the complete subsample, see Table 3), as expected for a lowdensity environment. On the other hand, we find evidence that spiral galaxies also increase their rate of radio activity with environment. The ratios for the spirals in our sample of isolated galaxies are clearly lower than for samples in denser environments.

\section{Conclusions}

In this paper, we have studied the rate of AGN candidates in a well-defined complete sample of isolated galaxies as part of the AMIGA project. We have focussed on two methods that make use of the radio (NVSS) and FIR (IRAS) data for our sample, and complemented them with additional data found in the literature. Our main results for the AMIGA sample are:

- Our sample is mostly radio quiet, with most galaxies $(98.6 \%)$ having radio powers lower than $10^{23} \mathrm{~W} \mathrm{~Hz}^{-1}$, consistent with the high ratio of late-type galaxies in our sample.

- We have selected radio-excess candidate galaxies above the radio-FIR correlation for our complete subsample, and revised the results using FIRST data to exclude back/foreground sources. We find less than $0.8 \%$ radioexcess galaxies with an excess of a factor 5 and less than $4.0 \%$ for a lower excess of a factor 3 .

- Using the IRAS flux ratio $S_{25 \mu \mathrm{m}} / S_{60 \mu \mathrm{m}}$ to select AGN candidates we find a frequency of AGN candidates of $\backsim 28 \%$ with a lower limit of $\sim 7 \%$.

- From NED and the Véron-Cetty catalogues, we found $n=29$ AGN candidates (including LINERs and NLAGN).

- The final catalogue contains a total of 89 AGN candidates. This catalogue is available in electronic form at the $\mathrm{CDS}^{2}$ and at the AMIGA web page ${ }^{3}$.

We have compared our results with those found in the literature and interpreted them, taking into account that our sample was

\footnotetext{
2 ftp://cdsarc.u-strasbg.fr/

3 http://www.iaa.csic.es/AMIGA.html
} 
selected using optical criteria and that we used a well-defined criterion of isolation. We conclude that:

- The AMIGA sample has the lowest ratio of AGN candidates, both globally and considering early-type and late-type galaxies separately.

- Field galaxies as well as galaxies in poor cluster and group environments (e.g., outer parts of clusters) show intermediate values, although the numbers are only illustrative as numerous selection effects affect the conclusions. The outer parts of clusters seem as efficient as field/poor environments to trigger radio emission in $\mathrm{AGN}$.

- The highest rates of AGN candidates are found in the central parts of clusters, but also in pair/merger dominated samples.

- For all environments, a higher ratio of radio excess is found for early-type galaxies, as can be expected since massive ellipticals are the usual hosts for powerful radio jets.

- Both elliptical and spiral galaxies increase their radio excess activity for denser environments. This increment supports that the density-morphology relation is not the only explanation for the enhancement in AGN frequency in denser environments, i.e., nuclear activity is not only associated with dense environments due to its higher content in ellipticals, but is directly triggered by it.

Hence, the environment seems to play a crucial role in the development of nuclear activity both at large scales and in strong one-to-one interactions.

Finally, we notice that the AMIGA sample appears to represent the most nurture-free population of luminous early-type galaxies as confirmed by their lack of radio excess above the FIR-radio correlation. The catalogue presented here can be used as a baseline for forthcoming studies about the relation between environment and nuclear activity. We plan to complement it with optical spectroscopy data.

Acknowledgements. We would like to warmly thank everybody who provided data for this study. The authors would also like to acknowledge the anonymous referee for his/her suggestions to improve the paper.

L.V.M., U.L., S.L., J.S. and J.S.M. are partially supported by DGI Grant AYA 2005-07516-C02-01 and Junta de Andalucía (Spain). S.L. was partially supported by an Averroes fellowship contract from the Junta de Andalucía. UL is supported by a Ramon y Cajal fellowship contract and by the DGI Grant ESP2003-00915. J.S.M. is supported by a fellowship from the Secretaría de Estado de Educación y Universidades.

This research has made use of the NASA/IPAC Extragalactic Database (NED) which is operated by the Jet Propulsion Laboratory, California Institute of Technology, under contract with the National Aeronautics and Space Administration.

\section{References}

Alonso, M. S., Lambas, D. G., Tissera, P., \& Coldwell, G. 2007, MNRAS, 375, 1017

Andersen, V. \& Owen, F. N. 1995, AJ, 109, 1582

Bertin, E., \& Arnouts, S. 1996, A\&AS, 117, 393

Bushouse, H. A. 1986, AJ, 91, 255

Condon, J. J., Anderson, M. L., \& Helou, G. 1991, ApJ, 376, 95

Condon, J. J., \& Broderick, J. J. 1991, AJ, 102, 1663
Condon, J. J., Anderson, E., \& Broderick, J. J. 1995, AJ, 109, 2318

Condon, J. J., Cotton, W. D., \& Broderick, J. J. 2002, AJ, 124, 675

Corbett, E. A., Norris, R. P., Heisler, C. A., et al. 2002, ApJ, 564, 650

Coziol, R., Iovino, A., \& de Carvalho, R. R. 2000, AJ, 120, 47

Dahari, O. 1985, AJ, 90, 1772

de Grijp, M. H. K., Miley, G. K., Lub, J., \& de Jong, T. 1985, Nature, 314, 240

Drake, C. L., McGregor, P. J., Dopita, M. A., \& van Breugel, W. J. M. 2003, AJ, 126,2237

Drake, C. L., McGregor, P. J., \& Dopita, M. A. 2004, AJ, 128, 955

Dultzin-Hacyan, D., Krongold, Y., Fuentes-Guridi, I., \& Marziani, P. 1999, ApJ, 513, L111

Fuentes-Williams, T., \& Stocke, J. T. 1988, AJ, 96, 1235

González-Martín, O., Masegosa, J., Márquez, I., Guerrero, M. A., \& DultzinHacyan, D. 2006, A\&A, 460, 45

Helou, G., Khan, I. R., Malek, L., \& Boehmer, L. 1988, ApJS, 68, 151

Helou, G., Soifer, B. T., \& Rowan-Robinson, M. 1985, ApJ, 298, L7

Ho, L. C. 1999, Adv. Space Res., 23, 813

Ho, L. C., \& Ulvestad, J. S. 2001, ApJS, 133, 77

Hooper, E. J., Impey, C. D., Foltz, C. B., \& Hewett, P. C. 1995, ApJ, 445, 62

Impey, C., \& Gregorini, L. 1993, AJ, 105, 853

Isobe, T., Feigelson, E. D., \& Nelson, P. I. 1986, ApJ, 306, 490

Isobe, T., Feigelson, E. D., Akritas, M. G., \& Babu, G. J. 1990, ApJ, 364, 104

Karachentseva, V. E. 1973, Astrofizicheskie Issledovaniia Izvestiya Spetsial'noj Astrofizicheskoj Observatorii, 8, 3

Kellermann, K. I., Sramek, R., Schmidt, M., Shaffer, D. B., \& Green, R. 1989, AJ, 98, 1195

Krongold, Y., Dultzin-Hacyan, D., \& Marziani, P. 2003, in Rev. Mex. Astron. Astrofis. Conf. Ser., 105

Laurikainen, E., \& Salo, H. 1995, A\&A, 293, 683

Leon, S., \& Verdes-Montenegro, L. 2003, A\&A, 411, 391

Leon, S., Verdes-Montenegro, L., Sabater, J., et al. 2008, A\&A, 485, 475

Lisenfeld, U., Verdes-Montenegro, L., Sulentic, J., et al. 2007, A\&A, 462, 507

Lisenfeld, U., Voelk, H. J., \& Xu, C. 1996, A\&A, 314, 745

MacKenty, J. W. 1989, ApJ, 343, 125

MacKenty, J. W. 1990, ApJS, 72, 231

Marcum, P. M., Aars, C. E., \& Fanelli, M. N. 2004, AJ, 127, 3213

Martinez, M. A., del Olmo, A., Focardi, P., \& Perea, J. 2006a, ArXiv Astrophysics e-prints

Martinez, M. A., del Olmo, A., Perea, J., \& Coziol, R. 2006b, ArXiv Astrophysics e-prints

Mauch, T., \& Sadler, E. M. 2007, MNRAS, 375, 931

Miller, C. J., Nichol, R. C., Gómez, P. L., Hopkins, A. M., \& Bernardi, M. 2003, ApJ, 597, 142

Miller, N. A., \& Owen, F. N. 2001, AJ, 121, 1903

Niklas, S. 1997, A\&A, 322, 29

Niklas, S., Klein, U., \& Wielebinski, R. 1995, A\&A, 293, 56

Omar, A., \& Dwarakanath, K. S. 2005, A\&A, 26, 89

Petrosian, A. R. 1982, Astrofizika, 18, 548

Rafanelli, P., Violato, M., \& Baruffolo, A. 1995, AJ, 109, 1546

Reddy, N. A., \& Yun, M. S. 2004, ApJ, 600, 695

Roy, A. L., Norris, R. P., Kesteven, M. J., Troup, E. R., \& Reynolds, J. E. 1998, MNRAS, 301, 1019

Sadler, E. M., Jackson, C. A., Cannon, R. D., et al. 2002, MNRAS, 329, 227

Schmitt, H. R. 2001, AJ, 122, 2243

Schmitt, H. R., Ulvestad, J. S., Antonucci, R. R. J., \& Kinney, A. L. 2001, ApJS, 132,199

Schmitt, J. H. M. M. 1985, ApJ, 293, 178

Shimada, M., Ohyama, Y., Nishiura, S., Murayama, T., \& Taniguchi, Y. 2000, AJ, 119, 2664

Sopp, H. M., \& Alexander, P. 1991, MNRAS, 251, 14P

Sulentic, J. W., Verdes-Montenegro, L., Bergond, G., et al. 2006, A\&A, 449, 937

Van Duyne, J., Beckerman, E., Salzer, J. J., et al. 2004, AJ, 127, 1959

Verdes-Montenegro, L., Sulentic, J., Lisenfeld, U., et al. 2005, A\&A, 436, 443

Verley, S., Leon, S., Verdes-Montenegro, L., et al. 2007a, A\&A, 472, 121

Verley, S., Odewahn, S. C., Verdes-Montenegro, L., et al. 2007b, A\&A, 470, 505

Véron-Cetty, M.-P., \& Véron, P. 2006, A\&A, 455, 773

Yun, M. S., Reddy, N. A., \& Condon, J. J. 2001, ApJ, 554, 803 